\title{
MUTUAL DIFFUSION COEFFICIENT OF AQUEOUS SUGAR SOLUTIONS
}

\author{
YUJI SANO* AND SHUICHI YAMAMOTO \\ Department of Applied Chemistry and Chemical Engineering, \\ Yamaguchi University, Ube, 755
}

Key Words: Food Chemical Engineering, Mass Transfer, Mutual Diffusion Coefficient, Sugar, Maltodextrin, Aqueous Solution, Drying

\begin{abstract}
The mutual diffusion coefficient of aqueous solutions with sugars of various molecular weight. obtained by drying experiment of the solution. was investigated for the effects of concentration. temperature and molecular weight of sugar.

The mutual diffusion coefficient of dilute solution was correlated using Wilke's diffusion factor. The concentration dependence was presented by linear relation of the logrithm of the diffusion coefficient vs. the sugar mole fraction regardless of temperature. The slopes of the linear relations were expressed by a power function of the molecular weight. From the linear relation. the activation energy was expressed also by linear relation of the sugar mole fraction. in which the slopes were expressed by the other power function of the molecular weight. The resultant estimation equation covers well for the temperature range 298-323 $\mathrm{K}$ and for the molecular weight range of sugars. from 180 for glucose to 6100 for maltodextrin.
\end{abstract}

\section{Introduction}

Analysis of the drying process for food materials such as aqueous solutions containing sugars skim milk, coffee extract, etc., and solid foods such as potato, apple tissue macaroni etc., depends on a knowlege of the diffusion coefficients ${ }^{4)}$. Sugars are essential in various foods and also play an important role in biochemical and medical products such as dry binder, thickening agents, stabilizing agents for enzymes. In an aqueous solution of sugars the diffusion coefficient usually shows strong concentration dependence.

The mutual diffusion coefficients of aqueous solutions for various sugars have been reported by several investigators, such as those of glucose (Gladden and Dole $^{3)}$ ) sucrose (Ellerton and Dunlop ${ }^{2)}$ ), $\mathrm{x}$ and $\mathrm{y}$ lose and maltose (Ueadaira and Uedaira ${ }^{8)}$ ). But most of these data are limited to room temperature, and the dependences on concentration and temperature have not been investigated widely. Systematic surveys of the effect of sugar molecular weight are also few.

The authors ${ }^{10)}$ measured the diffusion coefficients of various sugars by the capillary cell method in dilute solution and by the drying experiment of aqueous solution in concentrated range. Simple empirical equations for the dependence of concentration and temperature were then derived, without taking account of the effect of molecular weight.

Here. based on the previous data ${ }^{10)}$, the effect of sugar molecular weight on the mutual diffusion coefficient is surveyed and estimation equations are derived as a function of concentration, temperature and the molecular weight of the sugar.

\footnotetext{
* Received April 23, 1993. Correspondence concerning this article should be addressed to Y. Sano.
}

\section{Data of Diffusion Coefficient}

The experimental apparatus and the procedures were deseribed in a previous report ${ }^{10}$. Sugars investigated in this study are monosaccharide (glucose), disaccharides (sucrose, maltose, lactose) and oligosaccharides (maltodextrin of various $D E$ value) as shown in Table 1.

The diffusion coefficients in the concentrated range were calculated as a function of concentration from the previous drying rate data obtained for various sugar aqueous solutions, using the direct calculation method for the regular regime given by the authors ${ }^{6}$, assuming zero surface concentration or a certain finite value of surface concentration corresponding to the effective zero drying rate. In the calculating range of concentration (average water content larger than $0.15 \mathrm{~kg}$ water $/ \mathrm{kg}$ sugar for the drying rate), in which the linear relation of $\log D$ vs. $X_{s}$ was obtained through all sugars investigated as shown later, the calculation assuming zero surface concentration was convenient and satisfactory. The assumption of zero surface concentration corresponds to the supersaturated conditions of the solution near the surface in the early stage of the regular regime. In the drying experiments of the sugar solutions listed in the Table 1, transparancy of the solution was maintained and no precipitation was found during drying. The method of calculating the diffusion coefficient proposed by Schoeber ${ }^{7)}$ was also tested and close agreement with the results by the method of the authors ${ }^{6}$ ) was confirmed.

\section{Results and Discussion}

\subsection{The diffusion coefficient in dilute solution}

The mutual diffusion coefficient in dilute aqueous solution for the various sugars at $303 \mathrm{~K}$, obtained by the capillary cell method, are shown in Table 1 . They are 
Table 1. Sugars investigated in this study. $D_{0}$ is the observed value by the capillary-cell method $^{10)}$

\begin{tabular}{lcc}
\hline \multicolumn{1}{c}{ Sugars } & $M_{\mathrm{s}}[\mathrm{kg} / \mathrm{kmol}]$ & $D_{0}\left[\mathrm{~m}^{2} / \mathrm{s}\right] * 10^{10}$ \\
\hline glucose & 180 & 7.0 \\
sucrose & 342 & 6.0 \\
maltose & 342 & 6.0 \\
lactose & 342 & 6.0 \\
maltotriose & 504 & 4.3 \\
maltopentaose & 828 & 3.4 \\
oligotose & 570 & 5.1 \\
maltodextrin $(D E=40)$ & 910 & 3.8 \\
maltodextrin $(D E=25)$ & 1300 & 2.9 \\
maltodextrin $(D E=11)$ & 6100 & 2.2 \\
\hline
\end{tabular}

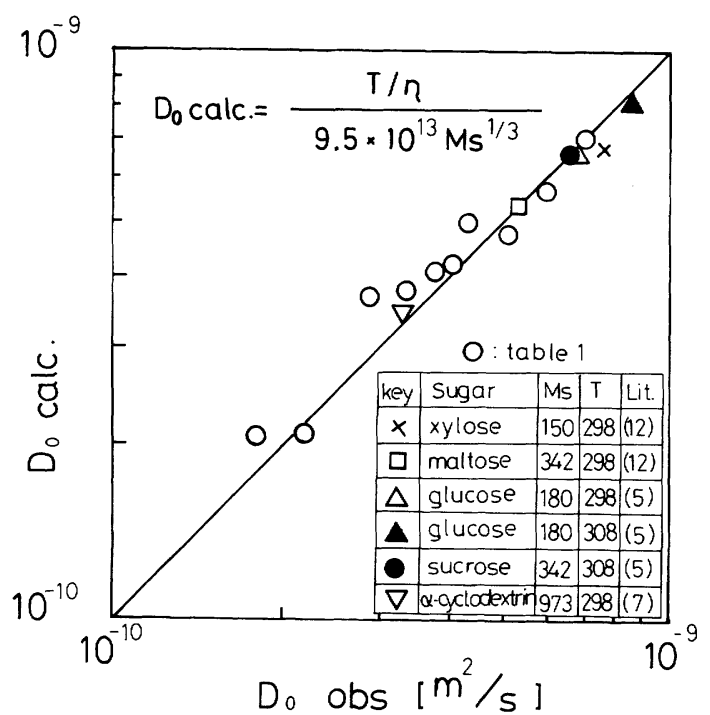

Fig. $1 D_{0}$ by Eq. (1) in comparison with observed values ${ }^{10)}$

correlated using the diffusion factor by Wilke and Chang ${ }^{9}$. Molar volume is calculated from the molecular weight of sugar by $V=M_{s} / d_{s}$, assuming a constant density of sugars $d_{s}=1560 \mathrm{~kg} / \mathrm{m}^{3}$ (density of glucose). An empirical equation with the term of molecular weight of solute $M_{s}$ is then presented as follows.

$$
\frac{T}{D_{0} \eta}=9.5 * 10^{13} M_{s}^{1 / 3}
$$

where $\eta$ is the viscosity of water at temperature $T$. Eq. (1) presents well the values of $D_{0}$ in Table 1 and the values of various sugars in the literatures ${ }^{3,5,8)}$, as shown in Fig. l.

\subsection{Concentration dependence of the diffusion coefficient}

In the drying experiment with sugar aqueous solu$\operatorname{tion}^{10)}$. the temperature of the solution reached the drying air temperature in the later drying stage of the regular regime. The diffusion coefficients were calculated as a function of water content from the regular regime drying-rate curves at the drying air temperature, using the methods by authors ${ }^{6)}$ in the range of average water content $(u>0.15)$.

The diffusion coefficient in the concentrated range

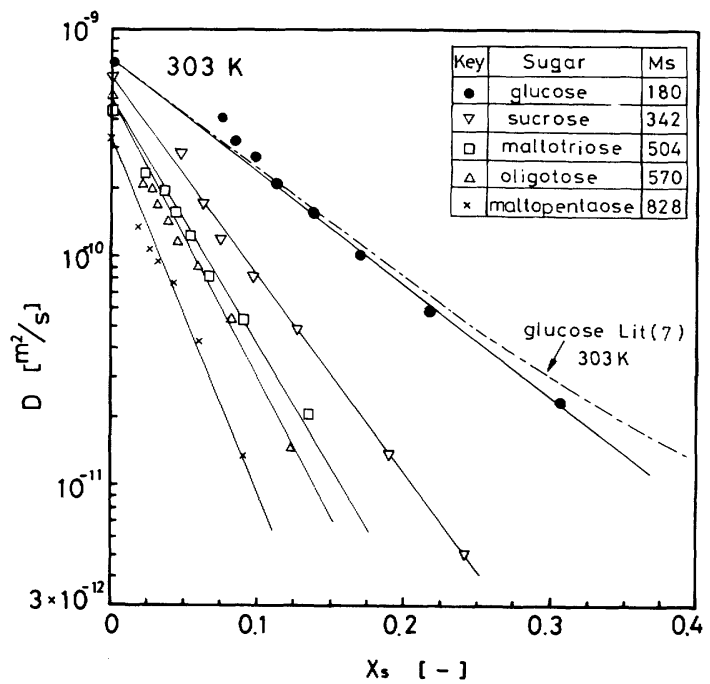

Fig. 2 Concentration dependence $D$ vs. $X_{s}$ for various sugars at $303 \mathrm{~K}$

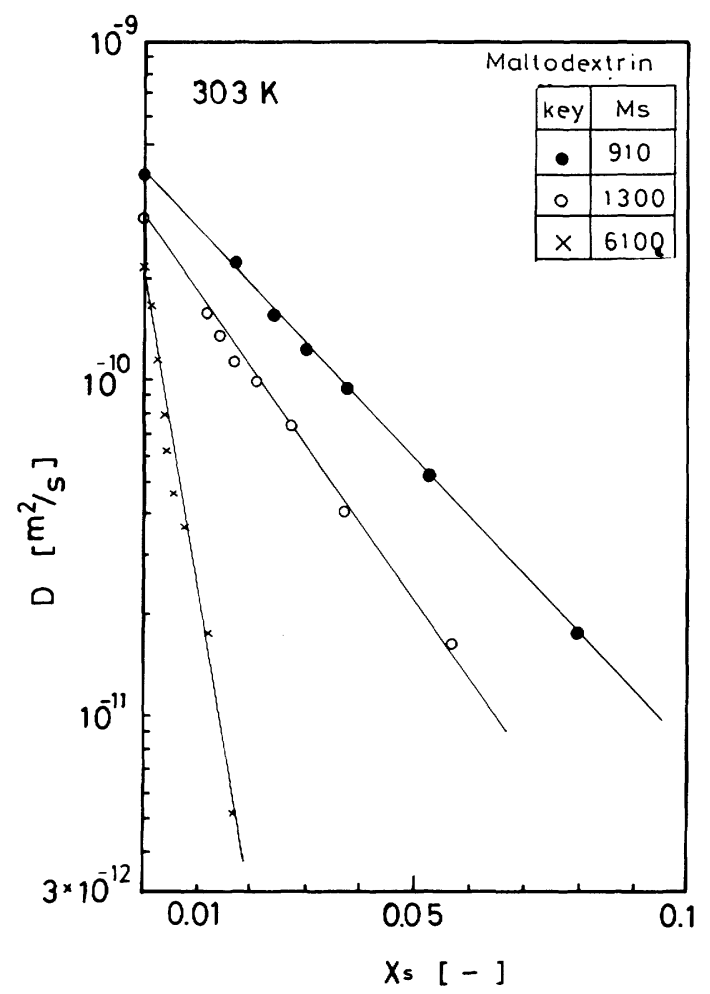

Fig. 3 Concentration dependence $D$ vs. $X_{s}$ for various maltodextrins at $303 \mathrm{~K}$

calculated from the drying rate data are correlated with the mole fraction of solute.

$$
X_{s}=\frac{1}{1+u\left(M_{s} / M_{w}\right)}
$$

The plots of $\log D$ vs. $X_{s}$ for the various sugar solutions are presented as linear relations in Figs. 2 and $\mathbf{3}$. The linear relations reached the values of $D_{0}$ calculated from Eq. (1) at $X_{s}=0$ quite well for the various sugars.

From the fact of the linear relation for the concentration dependence of the diffusion coefficient, the correlation equation is derived using the slope $\alpha$ of $\log D$ vs. 


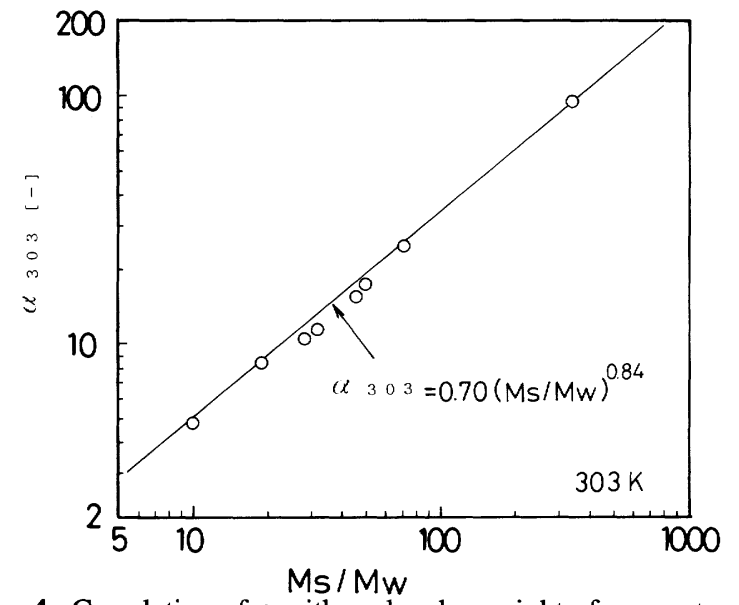

Fig. 4 Correlation of $\alpha$ with molecular weight of sugar at $303 \mathrm{~K}$

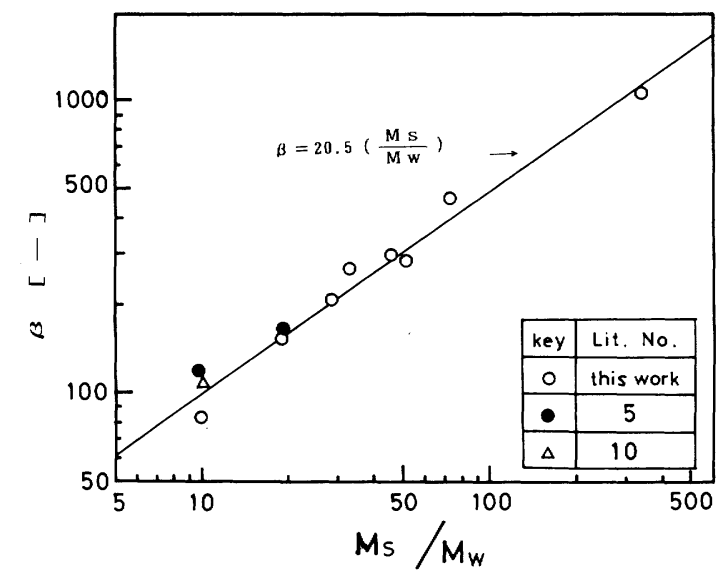

Fig. 5 Correlation of $\beta$ with molecular weigllt of sugar

$X_{s}$

$$
\log \left(D / D_{0}\right)=-\alpha X_{s}
$$

The values of $\alpha$ for various sugar solutions at $303 \mathrm{~K}$ are correlated with the molecular weight ratio of solute and solvent (water) as shown in Fig. 4. The correlation equation of $\alpha$ for $303 \mathrm{~K}$ is presented as follows.

$$
\alpha_{303}=0.70\left(M_{s} / M_{w}\right)^{0.84}
$$

\subsection{Correlation equation of diffusion coefficient}

If the linear relation of $\log D$ vs. $X_{s}$ by Eq. (3) holds at any temperature, then the slope $\alpha_{T}$ at temperature $T$ is related to the slope $\alpha_{303}$ at $303 \mathrm{~K}$, using the activation energy as follows.

$$
\begin{aligned}
\ln \left(\frac{D_{0, T}}{D_{0,303}} \cdot \frac{D_{303}}{D_{T}}\right) & =2.303\left(\alpha_{T}-\alpha_{303}\right) X_{s} \\
& =\frac{\Delta E-\Delta E_{0}}{R}\left(\frac{1}{T}-\frac{1}{303}\right)
\end{aligned}
$$

The activation energy should be expressed by the following linear relation of the sugar mole fraction $X_{s}$, which corresponds to the constancy of the slope $\alpha$ of Eq. (3), regardless of temperature.

$$
\Delta E-\Delta E_{0}=\beta X_{s}
$$

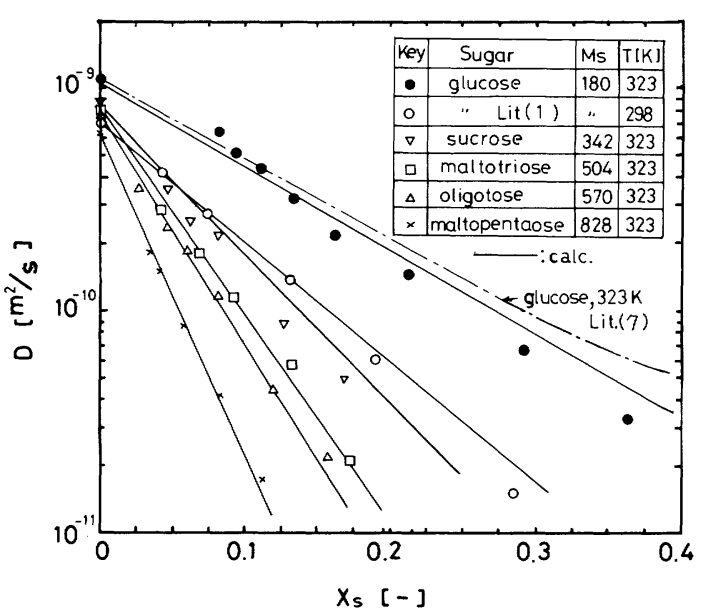

Fig. 6 Comparison of $D$, observed and calculated, for various sugars

Using $\beta, \alpha_{T}$ is expressed as follows.

$$
\alpha_{T}=\alpha_{303}+\frac{\beta}{2.303 R}\left(\frac{1}{T}-\frac{1}{303}\right)
$$

The activation energy $\Delta E_{0}$ for the dilute solution $\left(X_{s}=\right.$ 0 ) is evaluated from Eq. (1), using the temperature change of water viscosity as $17.3 \mathrm{~kJ} / \mathrm{mol}$.

The values of $\beta$ for various sugars were calculated by Eq. (7) and correlated with the molecular weight of sugar, as shown in Fig. 5. The correlation equation of $\beta$ is expressed as follows.

$$
\beta=20.5\left(M_{s} / M_{w}\right)^{0.7}
$$

Then the activation energy is

$$
\Delta E=17.3+20.5\left(M_{s} / M_{w}\right)^{0.7} X_{s}
$$

and $\alpha$ at temperature $T$ is

$$
\alpha_{T}=\alpha_{303}+20.5 \frac{\left(M_{s} / M_{w}\right)^{0.7}}{2.303 R}\left(\frac{1}{T}-\frac{1}{303}\right)
$$

From Eqs. (1), (3), (4) and (10), the values of $D$ are calculated and are compared with the experimental values for various sugars at $323 \mathrm{~K}$ and reported values in the literature such as those of glucose at $298 \mathrm{~K}^{1)}$ and 323 $\mathrm{K}^{7)}$ in Fig. 6. The same comparisons are shown for maltodextrins in Fig. 7. The linear $\operatorname{lines}$ of $\log D$ vs. $X_{s}$. calculated with the slope by Eq. (10) and with the values of $D_{0}$ by Eq. (1), fit the experimental values and literature values quite satisfactorily. Thus the estimation equations derived here will be useful in predicting the mutual diffusion coefficient of aqueous sugar solutions, in the concentration range in which the linear relation of $\log D$ vs. $X_{s}$ holds. The error of estimation will be less than $30 \%$, as shown in Figs. 2, 3, 6 and 7.

\section{Conclusions}

The concentration-dependent mutual diffusion coefficient of sugar aqueous solutions are investigated from glucose to maltodextrin (molecular weight $=6100$ ) 


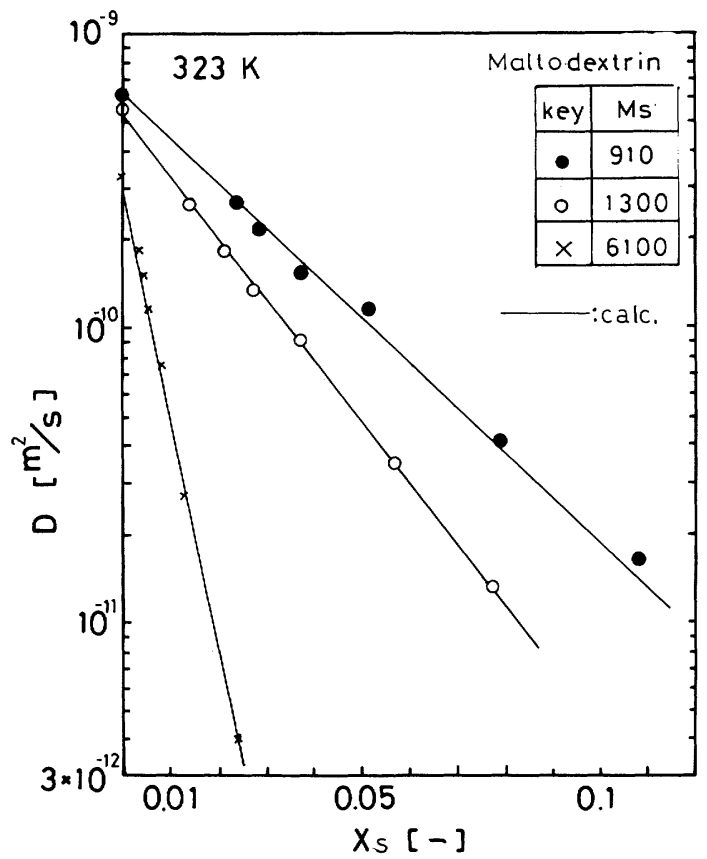

Fig. 7 Comparison of $D$, observed and calculated, for various maltodextrins

for the temperatures up to $50^{\circ} \mathrm{C}$. In the range of water content larger than $0.15 \mathrm{~kg}$ water $/ \mathrm{kg}$ sugar, the concentration dependence is expressed by linear relations of the logarithm of the diffusion coefficient vs. the mole fraction of sugar, regardless of temperature. Using the slope of the linear relation, empirical equations, Eqs. (1), (3), (4) and (10), for estimation of the diffusion coefficient are derived as a function of concentration, temperature and molecular weight of sugar. The error of the estimation may be within $30 \%$ in the range considered.

\section{Acknowledgement}

This work was financially supported by a Grant-in-Aid for Scientific Research from the Ministry of Education Science and Culture.

\section{Nomenclature}

$D \quad=$ mutual diffusion coefficient $\left[\mathrm{m}^{2} / \mathrm{s}\right]$

$D_{0} \quad=D$ of dilute solution

$D E \quad=$ dextrose equivalent

$\Delta E \quad$ = activation energy of diffusion coefficient

$M_{s} \quad=$ molecular weight of solute

$M_{w} \quad=$ molecular weight of solvent

$R \quad=$ gas constant

$u \quad=$ water content

$T \quad=$ temperature

$X_{s} \quad=$ mole fraction of solute

$\alpha \quad=$ slope of $\log D$ vs. $X_{s}$

$=$ slope of $\Delta E$ vs. $X_{s}$

$=$ viscosity of water

Literature cited

1) Chandrasekaran, S.K. and C. Judson King: AIChE J., 18, p. 513520 (1972)

2) Ellerton, H.D. and P. Dunlop: J. Phys. Chem., 71, p. 1291-1297 (1967)

3) Gladden, J.K. and M. Dole: J. Am. Chem. Soc., 75, p. 3900-3904 (1953)

4) Luyben, K.Ch.A.M., J.J. Olieman and S. Bruin: Drying ' 80 , vol. 2, ed. by A.S. Mujumdar, Hemisphere, p. 233-243 (1980)

5) Paduano, L., R. Sartorio, V. Vitagliano, J.G. Albright, D.G. Miller and J. Mitchell: J. Phys. Chem., 94, P. 6885-6888 (1990)

6) Sano, Y. and S. Yamamoto: J. Chem. Eng. Japan, 23, p. 331-338 (1990)

7) Schoeber, W.J.A.H.: Ph.D. Thesis, Tech. Univ. Eindhoven (1976)/Schoeber, W.J.A.H. and H.A.C. Thijssen: AIChE Symp. Series, 73 (163), p. 12-24 (1977)

8) Ueadaira, H. and H. Uedaira: Bulletin Chem.Soc. Japan, 42, p. 2140-2142 (1969)

9) Wilke C.R. and P. Chang: AIChE J., 1, p. 264-270 (1955)

10) Yamamoto, S. and Y. Sano: World Congress III of Chem. Eng., vol. II, 8b-152, p. 564-567, Tokyo (1986)/Sano, Y. and S. Yamamoto: Report of Grant-in-Aid for Scientic Research (C60550669), (1987) 